\title{
Participación de padres y/o representantes en las actividades escolares: un acercamiento desde la hermenéutica
}

\section{Participation of parents or representatives in school activities: an approach from hermeneutics}

\author{
Yosmar José Díaz Chacín 1,* \\ ${ }^{1}$ Universidad Rómulo Gallegos, Venezuela
}

\section{RESUMEN}

El propósito fue interpretar desde la óptica de los docentes la participación de los padres y/o representantes en las actividades escolares de los estudiantes de tercer año de la Escuela Técnica Industrial "Pedro Zaraza" mediante un acercamiento hermenéutico: dicho estudio se enmarcó dentro del paradigma postpositivista con enfoque cualitativo y empleó el método hermenéutico. Tuvo como informantes claves 2 docentes y 2 padres y/o madres. Para la aprehensión de la información se empleó la entrevista semiestructurada. Como reflexiones producto del análisis hecho a la información aportada por los informantes se agrega que en las posturas adoptadas por los principales actores de la trama educativa padres, representantes y docentes, se observa una visión reduccionista y rígida en contraste con las perspectivas integracionista que requiere del encuentro entre los padres y educadores para potenciar las influencias y promover el desarrollo del educando. La dificultad conceptual para propiciar la interacción y participación de la familia en las actividades escolares de las instituciones educativas no radica en la sensibilización de estas instituciones sino en el planteamiento de qué entiende cada una de las partes por participación en el encuentro interactivo y cómo hacerlo. En conclusión, no existe participación activa de los padres en el seguimiento de las actividades escolares, consideran que esta situación se vive en todas las instituciones educativas venezolanas; asimismo los padres y representantes asumen que la institución educativa es un espacio para el cuidado de los hijos.

Palabras claves: Educación, hermeneútica, participación en actividades escolares.

\section{ABSTRACT}

This research had as fundamental purpose interpreted from the perspective of teachers involvement of parents and/or representatives in the school activities for the third year students of the "Pedro Zaraza" Industrial Technical School through a hermeneutical approach: this study is framed within the postpositivist paradigm with qualitative approach and used the hermeneutic method.It had as key informants 2 teachers and 2 fathers and/or mothers had as key informants.for the apprehension of the information, the semi-structured interview was used. As reflections product of the analysis made to the information provided by the informants, it is added that in the positions adopted by the main actors of the educative plot parents, representatives and teachers a reductionist and rigid vision is observed in contrast with the integrationist perspective that requires the meeting between parents and educators to enhance the influences and promote the development of the student.the conceptual difficulty to encourage interaction and participation of the family in school activities of educational institutions does not lie in the awareness of these institutions but in the approach of what each of the parties understand by participating in the interactive meeting and how to do it.In conclusion,there is no active participation of parents in the monitoring of school activities, consider that this situation is experienced in all Venezuelan educational institutions; also the parents and representatives assume that the educational institution is a space for the care of the children.

Keywords: Education, hermeneutics, participation in school activities.

Historial del artículo:

Recibido, 12 de agosto de 2017; aceptado, 5 de diciembre de 2017; disponible en línea, 15 de enero de 2018

* Profesor en Educación Integral de la Universidad Pedagógica Experimental Libertador. Magister Scientiarum en Educación Mención Investigación Educativa de la Universidad Rómulo Gallegos de Venezuela.

Correo: lapatriaenroscio@hotmail.com

Este es un artículo de acceso abierto, licencia CC BY (https://creativecommons.org/licenses/by/4.0/) 


\section{INTRODUCCIÓN}

En Venezuela, al igual que en otros países latinoamericanos, la política educativa está orientada en los últimos años a definir directrices teóricas y operativas que posibiliten una concentración de esfuerzos en materia de información y desarrollo deseables, tales como, el consenso en materia educativa, mejorar la calidad del docente, reducción de la deserción y más y mejor participación de la sociedad civil, teniéndolos como factores que permitirán asegurar la calidad educativa; hecho que se traduce en la formación de un alumnado preparado y capacitado.

Por ello, la transformación educativa que actualmente vive el país exige el desarrollo de una sociedad participativa, capaz de construir un país soberano, centrado en la igualdad, la libertad y la justicia social. Es por ello que los sistemas se ven urgidos por la necesidad de dar respuestas que van más allá de la tradicional dedicación a la enseñanza del saber, tal como lo plantea la Organización de las Naciones Unidas para la Educación, la Ciencia y la Cultura (UNESCO, 2003), al exponer como una exigencia que la educación trate de dar respuestas adecuadas a las necesidades y demandas de la sociedad. En consecuencia, la educación de calidad deberá preparar a los estudiantes para que sean capaces de dar respuestas positivas y oportunas a las mismas.

Desde esta concepción humana, integral y progresista, cada ciudadano será constructor(a) de una sociedad que le permita participar, responder y corresponder en la creación de cambios que permitan el desarrollo regional y nacional. En este sentido, el nuevo modelo de Educación Bolivariana, plantea principios donde la escuela es transformadora de la sociedad, caracterizándose por ser participativa y democrática, donde cada persona que la conforma tiene inherencia para tomar decisiones, ejecutar y evaluar actividades escolares.

Fundamentándose en lo anteriormente expuesto, y tomando en consideración los señalamientos que se realizan en el Currículo Nacional Bolivariano, es un imperativo la comunicación permanente entre la escuela y la comunidad, a fin de propiciar aspectos no sólo de orden formativo o pedagógico, sino que debe ir más allá y abarcar aspectos sociológicos fundamentales, tales como, organización para la salud, para el desarrollo social, cultural y local sostenible. Es así, como la escuela se convierte en un agente clave para la transformación de cada ciudadano, donde se conjugan el ser, saber, hacer y convivir como modelo de desarrollo, concebido en la Constitución de la República Bolivariana de Venezuela.
Los planteamientos anteriores, llevan implícito que el éxito del proceso educativo es responsabilidad de todos los actores que hacen vida activa dentro del mismo, es decir, padres y/o representantes, estudiantes (as), docentes, directivos y sociedad civil. De allí la necesidad de crear mecanismos para llevar a cabo la integración de la escuela y la comunidad y por ende, lograr la participación de la familia como elemento esencial en la tarea de educar y enseñar a sus hijos.

En sintonía conceptual con lo precedente, (Albornoz 2009) expresa que la participación de los padres juega un papel esencial en la educación de sus hijos, pues deben ser miembros participativos junto al docente en la responsabilidad que tienen en colaborar con la enseñanza y el aprendizaje.

En este orden de consideraciones, se hace evidente que el docente debe desempeñarse como un promotor de la participación a través de la aplicación de técnicas comunales, desarrollo de actividades en el sitio o lugar donde está ubicado el plantel, que ayuden a propiciar la participación y cooperación de la familia en la identificación y solución de problemas y necesidades. De esta manera, se potencian los vínculos entre la escuela y la comunidad, creando un ambiente óptimo para la educación y el aprendizaje.

A este respecto, las afirmaciones de (Lovera 2000), adquieren pertinencia en la medida en que sostiene que la participación es una estrategia metodológica concebida en la escuela, para la escuela y los educandos, elaborada por el conjunto de actores de la acción escolar, incluida la participación de la familia y otros integrantes de la comunidad. No obstante, más allá de la posición del autor precitado, la participación debe ser un compromiso, un eje axiológico en donde la familia recupere su rol protagónico. En este sentido, la participación de la familia en las actividades escolares es primordial, porque ella como entorno social más cercano al estudiante, representa un valor compartido, produciéndose en su seno los primeros aprendizajes, permitiendo desarrollar la tenacidad, honestidad, responsabilidad e independencia de sus miembros. En este orden de afirmaciones, (Alberdi 2009), denota lo siguiente: "La familia sigue siendo el primer referente fundamental en la sociedad, jugando un papel relevante como elemento de cohesión social y de bienestar, fundamentada bajo el valor de la solidaridad que la sitúa bajo relaciones de igualdad, libertad y respeto" (P. 38).Como se aprecia, resulta imperativo que la familia se integre al proceso de aprendizaje de los estudiantes, por ser ellos los primeros y principales educadores de sus hijos. Por ello, resulta imprescindible unir la escuela y la familia, pues la naturaleza misma de la educación humana empieza en el hogar y continúa en la escuela. 
No obstante, aun cuando resulta tan evidente la necesidad de que la familia asuma una participación activa en las actividades escolares para el logro de una educación convivencial, tal como lo refiere el Proyecto Educativo Nacional (1999), la realidad es otra, por lo general, más que una aceptación, se percibe una incertidumbre entre los diferentes actores del hecho educativo.

Ante este escenario, surge esta investigación la cual es consecuente con la gran responsabilidad que envuelve en la actualidad el hecho educativo. De allí la necesidad de indagar acerca de la participación de los padres y representantes en las actividades escolares de los estudiantes de Tercer Año de la Escuela Técnica "Pedro Zaraza" en San Juan de los Morros, Estado Guárico, Venezuela.

Las situaciones en las que se describe el evento en estudio, invitan al autor a acercarse al fenómeno con el interés de aproximarse a posibles respuestas, a partir de la siguiente interrogante:

¿Qué significado e importancia tiene desde la óptica de los docentes, la participación de los padres y/o representantes en las actividades escolares?.

En correspondencia con la interrogante, se formuló el siguiente propósito de estudio.

Interpretar desde la óptica de los docentes, el significado e importancia de la participación de los padres y representantes en las actividades escolares de los estudiantes de Tercer Año de la Escuela Técnica Industrial "Pedro Zaraza" en San Juan de los Morros, Estado Guárico.

Esta investigación es de gran importancia y posee gran relevancia, pues, al involucrar a la familia en las actividades escolares, se estará profundizando y fortaleciendo la participación de la misma en el proceso educativo, con el fin de ofrecer herramientas para enfrentar los problemas que afectan la educación de sus hijos e ir creando las condiciones para construir colectivamente una articulación con sentido bien definido entre la escuela y el contexto comunitario.

Por otra parte, esta investigación cobra relevancia por los aportes que ella genera; por tal razón la misma se justifica desde varias perspectivas. Desde la perspectiva epistemológica, este estudio es válido porque responde a una gran aspiración del Sistema Educativo Venezolano, como es la integración de la escuela a la comunidad y viceversa.

Desde la perspectiva metodológica, este estudio se planteó una investigación que accede a la observación de un fenómeno que se suscita en la realidad de la educación venezolana, como es la falta o poca asistencia o interés de la familia en las actividades escolares de sus hijos y representados, de la cual se obtendrá una serie de hallazgos y recomendaciones que servirán de antecedentes a posteriores investigaciones.

En lo correspondiente a los estudios considerados como antecedentes, se revisaron los realizados por (Reyes 2001), quien presenta un trabajo referido a la Influencia del Rol Familiar en el Rendimiento Escolar de los Alumnos de la Tercera Etapa del Ciclo Combinado "Arturo Michelena", Calabozo. El estudio se enmarcó en una investigación de campo no experimental, con una muestra de 269 padres y representantes y 277 estudiantes de diferentes grados. Entre las conclusiones a las que llegó el autor, se tiene que existen diferencias de criterios entre los padres y/o representantes y alumnos, destacándose así la influencia de la familia en el rendimiento escolar de los alumnos. Razón por la cual recomendó hacer reuniones consecutivas de padres y/o representantes en la institución, con el fin de incentivarlos para que cumplan de forma eficiente su función de formadores y orientadores en el hogar $y$, por ende, en el binomio alumno-escuela.

En esta misma línea de pensamiento, (Tablante 2002) realizó un trabajo cuyo objetivo fue proponer un Programa de Escuela para Padres para facilitar la integración de la Familia en el Proceso Educativo de los Alumnos de la Escuela Básica "Andrés Bello" de Calabozo, Estado Guárico. Esta investigación se correspondió con un Proyecto Factible, apoyado en un trabajo de campo de carácter descriptivo, con una muestra de 17 docentes y 85 padres y/o representantes. Entre las conclusiones más relevantes destacan la opinión de los docentes encuestados al expresar que los padres y/o representantes nunca participan en las actividades escolares de sus hijos y/o representados.

Por su parte, (Malpica 2003) presenta un trabajo denominado Integración Familia-Escuela como alternativa para mejorar el rendimiento escolar de los alumnos de la Primera Etapa de la Unidad Educativa "Tarcisio Conde", en Guardatinajas del Estado Guárico. Metodológicamente, el trabajo se corresponde con un diseño de campo de carácter descriptivo bajo la modalidad de Proyecto Factible, utilizando como muestra a 60 padres y/o representantes, arrojando como conclusión más importante la necesidad de integrar a la familia y la escuela como medio para mejorar el rendimiento escolar de los estudiantes de esta institución en la primera etapa.

Igualmente, se reseña el estudio elaborado por (Medina 2004), al que denominó Programa de Integración Comunitaria como Medio para Optimizar la Participación de los Padres y/o Representantes en el Proceso Educativo de sus hijos de la U.E. "Virgen Milagrosa" de Calabozo, Estado Guárico. La metodología utilizada correspondió a la modalidad 
de Proyecto Factible, apoyada en una investigación de campo de carácter descriptivo. Se seleccionó una muestra formada por 135 padres y 15 docentes. Para recolectar los datos se usaron dos instrumentos, uno dirigido a los padres y otro a los docentes. La conclusión más importante a la que llega el estudio está relacionada con el distanciamiento de la comunidad con respecto a la escuela y lo cerrada de esta a la incursión de la comunidad en algunos asuntos de su quehacer diario, por lo que es viable la puesta en práctica de la propuesta.

Por último, se refiere el trabajo de (Chirinos 2006), titulado Programa para mejorar la participación de padres y representantes en la actividades pedagógicas, recreativas y culturales de la U.E. "Socorro Acosta de Sánchez". La metodología se correspondió con un proyecto factible. La población la conformaron quince docentes y cuatrocientos padres y representantes. El instrumento utilizado fue un cuestionario de preguntas cerradas. Entre las conclusiones del estudio se destaca el hecho de que se debe invertir tiempo, trabajo y recursos en la planificación de actividades que contribuyan al proceso integrativo de los padres y representantes.

Los estudios presentados, dejan ver la importancia de la participación de la familia en las actividades escolares, por cuanto se considera que la familia es responsable de la formación y del aprendizaje que se les imparte a sus representados a lo largo de toda su vida, consolidando el vínculo entre el hogar y la escuela como parte de una educación integrada establecida por el Estado como proyecto de país.

\section{MATERIAL Y MÉTODOS}

Esta investigación epistemológicamente hablando, se circunscribió dentro del denominado Paradigma de la Post-Modernidad o Post-Positivista, en donde a decir de Martínez (1999), se supera el esquema que considera la percepción como simple reflejo de las cosas reales y por tanto el conocimiento como copia de esa realidad. De allí que este estudio se sirvió de las herramientas de la investigación cualitativa, la cual, "permite el abordaje de la realidad estudiada a través de unas técnicas interpretativas que admiten describir, decodificar, traducir y sintetizar el significado, y no la frecuencia de hechos que ocurren más o menos naturalmente en el mundo social" (Ruiz e Ispizua, 1999).

Se asumió el método hermenéutico-dialéctico, propuesto por (Gadamer 1998). Se consideraron los siguientes momentos durante la investigación: Primer Momento: Incorporación del investigador al medio natural de los sujetos de estudio; incluyó el uso de técnicas para recopilar la información (registros, anotaciones, grabaciones). Segundo Momento: Audición de grabaciones, lecturas de transcripciones, anotaciones a margen de las mismas, lectura de registros de observaciones. Aquí se trató de pasar del dato a la vivencia misma de quien la produce, tal como dice (Dilthey citado en Moreno, 1945) "comprender significa pasar de la exterioridad de los datos a la vivencia originaria que los produce, sean gestos, lenguaje u objetos culturales en general".

Tercer Momento: Categorización de la información. Aquí se vació la información obtenida de las entrevistas. Cuarto Momento: Estructuración de la información mediante la cual se brinda una visión comprensiva de los eventos estudiados. Sexto Momento: Teorización para integrar la síntesis conceptual y las reflexiones.

El escenario donde se realizó la investigación, estuvo representado por la Escuela Técnica Industrial Robinsoniana "Pedro Zaraza", ubicada en la avenida "Luís Aparicio" de la ciudad de San Juan de los Morros, estado Guárico.

Se seleccionaron como informantes claves, dos (02) docentes, de la institución precitada y dos padres y/o madres o representantes. Se utilizó como técnica para recolectar la información la entrevista semiestructurada. El instrumento fue un guion de entrevista, un grabador y el propio investigador. El procesamiento de la información colectada, se realizó a través del uso de la categorización, la cual es una técnica que permite resumir o sintetizar en una idea o concepto, una palabra o expresión breve pero elocuente, es decir, un conjunto de información escrita, grabada o filmada para su fácil manejo posterior. En este sentido, se procedió a la categorización de la información recogida en función de los distintos eventos de interés en la investigación. Estas categorías serán descritas mediante la abstracción de significados para los principales eventos.Tal proceso de categorización es producto de la compenetración del investigador con el medio donde se recogen los hallazgos estructuración y triangulación. Tal como se observa en la Tabla $N^{\circ} 1$.

Las estructuras se derivan del proceso de categorización, y recogen el pensar y el sentir de cada uno de los informantes, lo que permite la comprensión y descripción del fenómeno estudiado en su contexto. El proceso de estructuración, consiste en el diseño de gráficos con la finalidad de mostrar datos cualitativos, correspondientes a cada uno de los informantes. Estas estructuras se derivan del proceso de categorización, y recogen el pensar y el sentir de cada uno de los informantes, lo que permite la comprensión y descripción del fenómeno estudiado en su contexto. (figura 1).

Se aprecia en la figura 1, que tanto docentes como padres y representantes, consideran que la 
Tabla 1

Categorización de las entrevistas, informante: Padre (Cactus)

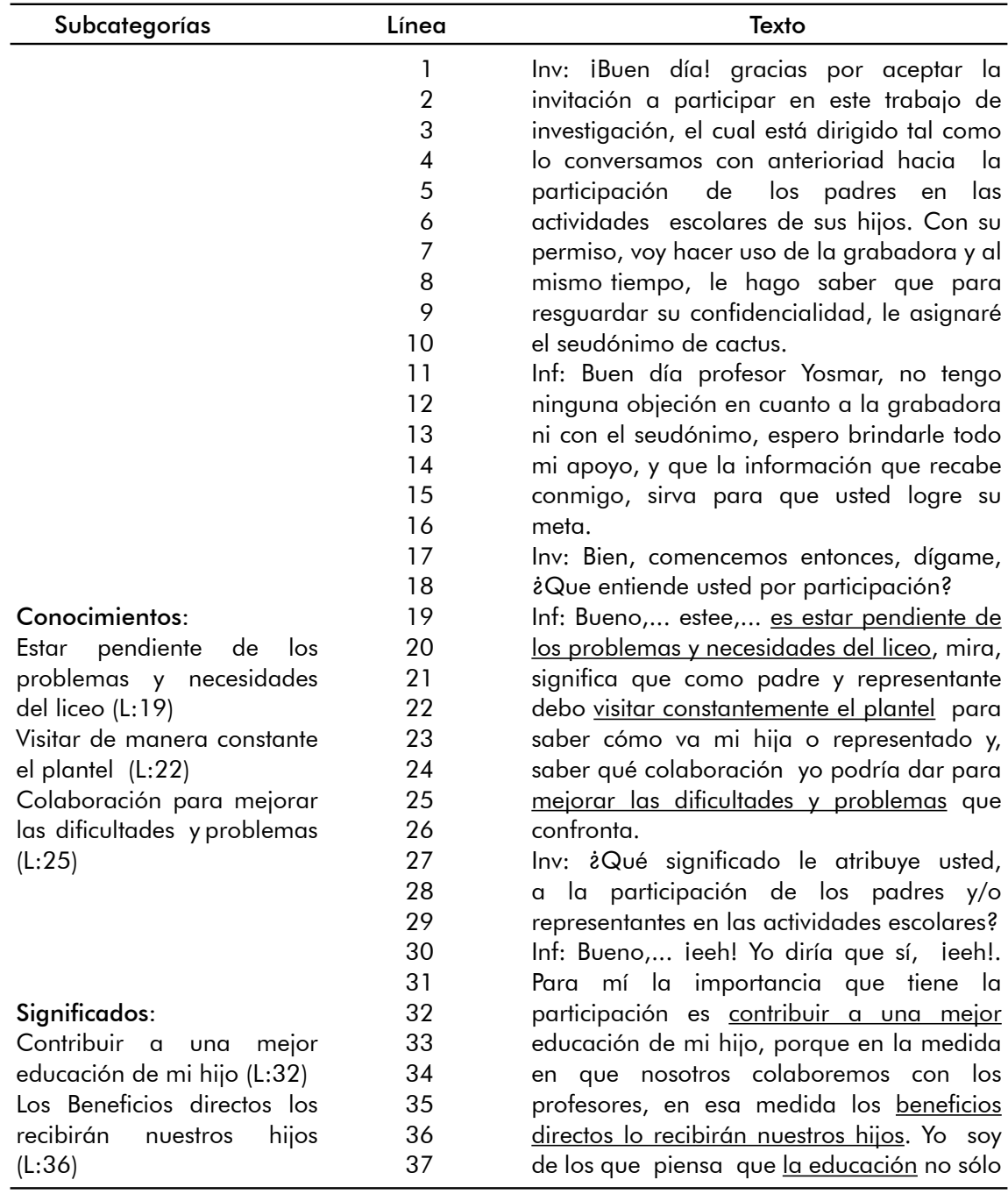

participación, es la manera mediante la cual los individuos actúan en una determinada actividad o proceso. A su vez, la piensan como un largo proceso de aprendizaje, actitudes y habilidades para intervenir en la toma de decisiones que afectan a todos los agentes que interactúan en el hecho educativo. Esto implica, estar visitando constantemente el plantel, para conocer las necesidades y así colaborar y prestar ayuda, para mejorar las dificultades y problemas que se puedan presentar.

En la figura 2, se evidencia según testimonio de los informantes, que no existe participación activa de los padres en el seguimiento de las actividades escolares, los entrevistados creen que esta situación se vive en todas las instituciones educativas. También, expresaron que los padres y representantes asumen la institución educativa como un espacio para el cuido de los hijos. De allí que, según ellos, hace falta mayor compromiso.
Otra técnica utilizada, fue la triangulación, la misma consiste en recoger y analizar datos desde distintos ángulos para compararlos y contrastarlos entre sí, es decir, que el investigador para corroborar la información obtenida, debe considerar la confluencia de diferentes fuentes de datos, perspectivas teóricas, investigadores y procedimientos metodológicos.

La triangulación, se utiliza para lograr la confirmabilidad de la información. De allí que la triangulación, es una técnica cuyo principio básico consiste en recoger y analizar datos desde distintos ángulos para compararlos y contrastarlos entre sí, es decir, que el investigador para corroborar la información obtenida, debe considerar la confluencia de diferentes fuentes de datos, perspectivas teóricas, investigadores y procedimientos metodológicos (tabla 2). 


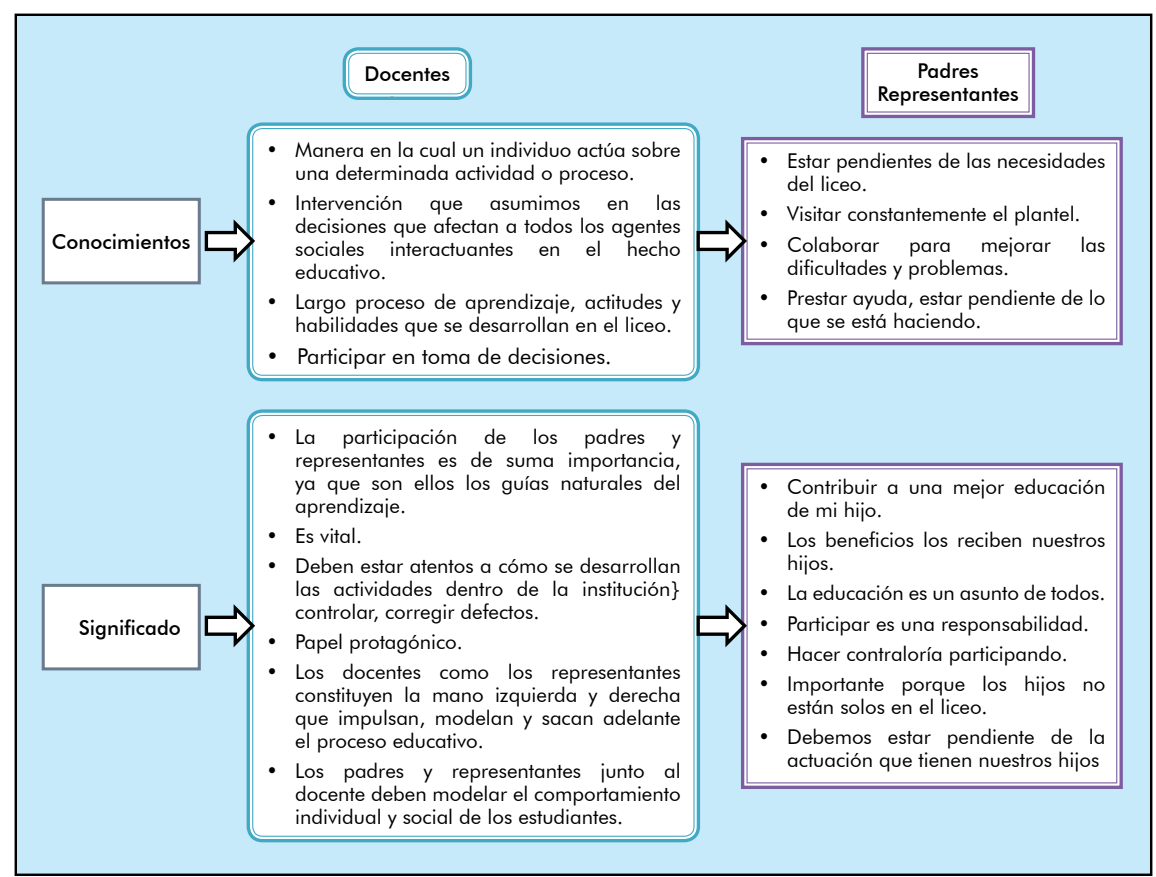

Figura 1. Estructura general con representación de las subcategorías, conocimientos y significado.

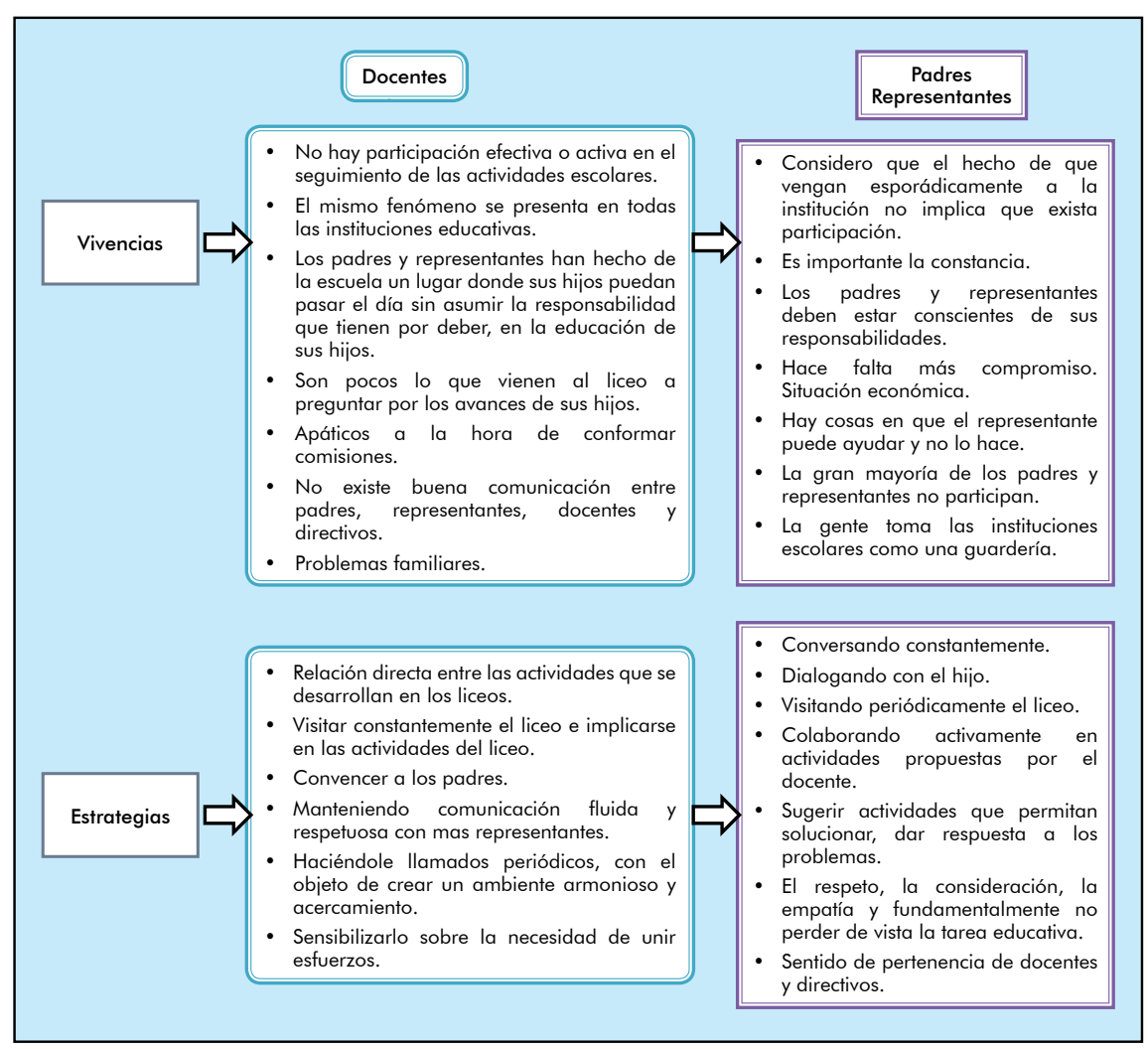

Figura 2. Estructura general con representación de las subcategorías, vivencias y estrategias. 
Tabla 2

Triangulación de la información.

\begin{tabular}{|c|c|c|c|}
\hline Subcategorías & $\begin{array}{c}\text { Informantes } \\
\text { Docentes/Padres }\end{array}$ & $\begin{array}{c}\text { Teoría } \\
\text { (Autores) }\end{array}$ & Investigador \\
\hline Conocimientos: & $\begin{array}{l}\text { Los informantes entrevistados, } \\
\text { tanto docentes como padres y } \\
\text { representantes, consideran que } \\
\text { la participación, es la manera } \\
\text { mediante la cual los individuos } \\
\text { actúan en una determinada } \\
\text { actividad o proceso. A su vez, } \\
\text { la consideran como un largo } \\
\text { proceso d aprendizaje, actitudes } \\
\text { y habilidades para intervenir } \\
\text { en la toma de decisiones que } \\
\text { afectan a todos los agentes } \\
\text { que interactúan en el hecho } \\
\text { educativo. Esto implica, estar } \\
\text { visitando constantemente } \\
\text { el plantel, para conocer las } \\
\text { necesidades y así colaborar y } \\
\text { prestar ayuda, para mejorar las } \\
\text { dificultades y problemas que se } \\
\text { puedan presentar. }\end{array}$ & $\begin{array}{l}\text { Davies (2001), expone que la } \\
\text { participación es un proceso de } \\
\text { aprendizaje, alfabetización y al } \\
\text { mismo tiempo, un instrumento } \\
\text { eficaz para la formación y } \\
\text { educación de los seres humanos. } \\
\text { este autor, a su vez afirma que } \\
\text { la negociación, el compromiso, } \\
\text { así como la toma de decisiones } \\
\text { son instrumentos claves de la } \\
\text { participación. }\end{array}$ & $\begin{array}{l}\text { La participación es la vía o } \\
\text { el medio para mejorar la } \\
\text { calidad educativa, mejorar } \\
\text { las relaciones entre todos los } \\
\text { actores sociales involucrados en } \\
\text { el hecho educativo, implicarse } \\
\text { en la toma de decisiones, } \\
\text { promover el interés y reforzar } \\
\text { la igualdad de oportunidades. } \\
\text { Por tanto, participar es tomar } \\
\text { parte actyiva en cada una de } \\
\text { las distintas fases que afectan el } \\
\text { funcionamiento de la institución } \\
\text { escolar ensu totalidad. De allí } \\
\text { que la participación implica } \\
\text { necesariamente, la implicación, } \\
\text { compromiso y colaboración en } \\
\text { las actividades escolares de la } \\
\text { institución donde cursan estudios } \\
\text { nuestros hijos. }\end{array}$ \\
\hline Sigr & $\begin{array}{l}\text { Los informantes consideran que } \\
\text { la participación de los padres } \\
\text { es de suma importancia, ya que } \\
\text { ellos son los guias naturales del } \\
\text { aprendizaje de los hijos. Por lo } \\
\text { tanto, la participación de los } \\
\text { mismos, contribuye a una mejor } \\
\text { educación de los estudiantes. } \\
\text { Por otra parte, expresan que la } \\
\text { educación es un asunto de todos, }\end{array}$ & $\begin{array}{l}\text { Navarro (2000), expresa que la } \\
\text { participación es una actividad } \\
\text { básica, fundamental e importante } \\
\text { paa el desarrollo de una } \\
\text { educación integral. Asimismo } \\
\text { expresa, que la participación es } \\
\text { un elemento activo y primordial } \\
\text { para la }\end{array}$ & $\begin{array}{l}\text { La completa y activa participación } \\
\text { de los padres y representantes } \\
\text { en las actividades escolares, es } \\
\text { crucial para lograr un cambio } \\
\text { sistemático, continuo, real y } \\
\text { productivo para asegurar así, } \\
\text { acciones pedagógicas que } \\
\text { beneficien a los estudiantes y } \\
\text { comunidad en general. }\end{array}$ \\
\hline
\end{tabular}

\section{RESULTADOS}

Los informantes entrevistados, tanto docentes como padres y representantes, consideran que la participación, es la manera mediante la cual los individuos actúan en una determinada actividad o proceso. A su vez, la consideran como un largo proceso de aprendizaje, actitudes y habilidades para intervenir en la toma de decisiones que afectan a todos los agentes que interactúan en el hecho educativo. Esto implica, estar visitando constantemente el plantel, para conocer las necesidades y así colaborar y prestar ayuda, para mejorar las dificultades y problemas que se puedan presentar.

Desde mi Hermeneusis como investigador, pienso que la participación es la vía o el medio para mejorar la calidad educativa, mejorar las relaciones entre todos los actores sociales involucrados en el hecho educativo, implicarse en la toma de decisiones, promover el interés y reforzar la igualdad de oportunidades. Por tanto, participar es tomar parte activa en cada una de las distintas fases que afectan el funcionamiento de la institución escolar en su totalidad. De allí que la participación envuelve necesariamente, la implicación, compromiso y colaboración en las actividades escolares de la institución donde cursan estudios nuestros hijos.

De igual forma, los informantes consideran que la participación de los padres es de suma importancia, ya que ellos son los guías naturales del aprendizaje de los hijos. Por lo tanto, la participación de los mismos, contribuye a una mejor educación de los estudiantes ello, la participación juega un papel protagónico y vital en el proceso educativo. Es por ello, que los padres deben participar en las actividades escolares.

No obstante, según lo expresado por los informantes, no existe participación activa de los padres en el seguimiento de las actividades escolares, consideran que esta situación se vive en todas las instituciones educativas venezolanas. También, expresan que los padres y representantes asumen que la institución educativa es un espacio para el cuido de los hijos. De allí, que según ellos, hace falta mayor compromiso, pues, se muestran apáticos al momento de formar comisiones de trabajo. Aunado a ello, consideran que no existe buena comunicación entre padres y representantes, docentes y directivos. En este sentido, 
consideran que la falta de participación de los padres y representantes, pudiera deberse entre otros factores a la situación económica por la que atraviesan y/o problemas familiares.

Se considera que la participación de padres y representantes en las actividades escolares, está influenciada por diversos factores, entre ellos destacan: el desinterés o desmotivación, la falta de información necesaria acerca de la necesidad y responsabilidad que tienen de ser coparticipes de la educación de sus hijos. A nivel educativo, evasión al compromiso y el desfase comunicacional entre padres, representantes y docentes.

\section{DISCUSIÓN}

La participación como elemento clave de la vida democrática se ha ido fortaleciendo en todos los ámbitos de la sociedad y, por lo tanto, también en el terreno de la educación. De allí que la participación en la gestión educativa requiere poder tomar parte activa en la elaboración y desarrollo del proceso educativo. Se entiende entonces que la participación de los padres, como agentes del medio educativo, es de suma importancia para favorecer al máximo el desarrollo del niño, niña y adolescente, a fin de mejorar y alcanzar las metas y objetivos propuestos por la educación, fundamentados en la Ley Orgánica de Educación.

En consonancia, la educación como un proceso social exige la participación activa de todos los sectores, en especial de la familia, entonces la participación de los padres y/o representantes, puede considerarse como una vía o medio para mejorar la calidad educativa. Por lo tanto, la participación implica compromiso y colaboración en las actividades educativas de la institución donde cursan estudio nuestros hijos. De allí que, en la actualidad, la participación de los padres en la institución escolar se ha adoptado como un criterio de calidad y garantía de eficiencia de la acción educativa.

Concatenando con las ideas anteriores, interesa destacar que la participación de los padres en la escuela es en sí misma una práctica del juego democrático ya que comprende unas técnicas y valores que constituyen la esencia del sistema democrático como son el respeto, la tolerancia, la libertad de expresión, la valoración de otras opiniones, la discusión y el debate constructivo que hacen posible una convivencia pacífica y enriquecedora.

En este orden de consideraciones la investigación se orientó a interpretar desde la óptica de los docentes, el significado e importancia de la participación de los padres y representantes en las actividades escolares de los estudiantes de Tercer Año de la Escuela Técnica Industrial "Pedro Zaraza" en San Juan de los Morros, Estado Guárico.

A este respecto, la participación implica compromiso y colaboración en las actividades escolares de la institución donde cursan estudio nuestros hijos. De allí que, en la actualidad, la participación de los padres en la institución escolar se ha adoptado como un criterio de calidad y garantía de eficiencia de la acción educativa.

En las posturas adoptadas por los principales actores de la trama educativa padres, representantes y docentes, se observa una visión reduccionista y rígida en contraste con las perspectivas integracionista que requiere del encuentro entre los padres y educadores para potenciar las influencias y promover el desarrollo del educando. La dificultad conceptual para propiciar la interacción y participación de la familia en las actividades escolares de las instituciones educativas no radica en la sensibilización de estas instituciones sino en el planteamiento de qué entiende cada una de las partes por participación en el encuentro interactivo y cómo hacerlo.

Sin embargo, si se entiende que la participación es un medio para mejorar la calidad educativa, mejorar las relaciones entre los distintos sectores de la comunidad educativa, implicarse en la toma de decisiones, trabajar en equipo. Saben que la función de la actual escuela no es meramente transmitir conocimientos, sino también formar ciudadanos competentes social y culturalmente, lo que convierte la tarea de educar en algo más complejo, que debe ser compartido por los docentes y familias conjuntamente.

A este complejo asunto se le añade variables tales como el nivel académico de los padres, aunque los resultados de investigaciones no apuntan a una relación directa, es decir que padres con un nivel de escolarización bajo pueden mostrar interés y colaborar con la educación escolar de sus hijos y cooperar en las actividades o manifestar poca implicación en los temas escolares de los hijos depositando toda la responsabilidad en los docentes. Lo mismo ocurre con padres con un nivel superior de enseñanza. Tampoco el nivel socioeconómico de estos correlaciona directamente con su integración en las actividades educativas de la escuela.

En sintonía conceptual y epistemológica con lo precedente, resulta interesante reseñar la valoración que realiza Sánchez (1999), sobre los diferentes modelos educativos acerca de la familia, al cualificar el comportamiento de los padres: En primer lugar, está el modelo racional que se caracteriza por una gestión jerárquica de la relación educativa con los hijos. En este modelo, los padres deciden sobre el futuro del niño, joven o adolecente, la disciplina, el 
orden, la sumisión a la autoridad son el fundamento de la comunicación padre-hijo que pasa a ser de naturaleza autoritaria. En cuanto a su relación con los docentes, con frecuencia esta categoría de padres desconfía de la labor de los primeros y las dificultades de comunicación pueden llegar a ser insalvables. De hecho, algunos padres de esta categoría adoptan una actitud contestataria porque no aceptan resoluciones educativas que proceden del docente.

En segundo lugar, en el modelo humanista, que se inscribe en las teorías de Roger y Maslow se le otorga al hijo un gran poder de decisión y, al mismo tiempo, se le permite expresar sus emociones, se facilita el aprendizaje según su punto de vista. La comunicación que establece padre-hijos es empática y el centro de su preocupación suele ser el futuro del hijo. La interacción de la familia con el profesor se caracteriza por la empatía y la expresión de las emociones; importará mucho la autogestión y los medios para que la persona sea ella misma siendo esta preocupación esencial en este modelo. Los padres suelen buscar, preferentemente, del saber del docente y, por lo tanto, se puede afirmar que existe poca interacción en la realidad.

Por último, el modelo simbiosinérgico tiene una doble acepción: la palabra "simbio" que supone una asociación recíproca entre dos o más seres vivos y la de "sinérgico" que corresponde a las acciones coordinadas entre ambos. Implica, por tanto, la puesta en común de recursos del saber hacer de las personas afectadas. En este modelo, los padres suelen establecer una relación de reciprocidad con su hijo reconociendo los derechos y deberes de cada uno, e intentando guiar al hijo sobre la base de su propia experiencia, en este sentido, el dialogo es el medio más comúnmente utilizado en la familia. En su relación con los maestros estas categorías de padres se enfrentan a ellos con una idea de interdependencia y de reciprocidad en el aprendizaje y desenvolvimiento de cada uno, y donde todos deben aprender de todos.

En sintonía con los señalamientos realizados anteriormente, y a manera de reflexión, resulta fundamental destacar que la participación de los padres y/o representantes en el proceso educativo de sus hijos(as), favorece el crecimiento de los niños, niñas, jóvenes y adolescentes, y facilita un aprendizaje de calidad.

\section{REFERENCIAS BIBLIOGRÁFICAS}

Alberdi, I.; Escario, P. (2007), Los hombres jóvenes y la paternidad, Bilbao, Fundación BBVA.

Albornoz, O. (2009). La Familia y la Educación del Venezolano. U.C.V. Ediciones de la Biblioteca de Caracas.

Chirinos, J. (2006). Programa para mejorar la Participación de Padres y representantes en las Actividades Pedagógicas Recreativas y Culturales. Trabajo de Grado de Maestría no publicado. Universidad Bicentenaria de Aragua, Maracay Estado. Aragua.

Davies, D. (2001). Los padres en la escuela. Barcelona. Laia.

Gadamer, H (1998). Verdad y Método: Fundamentos de una Hermenéutica Filosófica. Salamanca.

Lovera, B. (2000). Programa de Escuela para Padres dirigido a mejorar las Relaciones Interpersonales Padres e Hijos en el Liceo Don Rómulo Gallegos, Apure. UPEL, Maracay, Trabajo de Maestría.

Malpica, A. (2003). Programa de Integración Familia-Escuela como Alternativa para Mejorar el Rendimiento Escolar en los Alumnos de la Tercera Etapa de la U.E. Francisco Conde, Guardatinajas. UNERG. Tesis de Grado de Maestría.

Martínez, M. (1999). La nueva Ciencia su Desafió, Lógica y Método. Editorial Trillas México.

Medina, L. (2004). Programa de Integración Comunitaria como Medio para Optimizar la participación de los Padres y Representantes en el Proceso Educativo de la U.E. Virgen Milagrosa de Calabozo, Estado Guárico. UNERG. Tesis de Grado de Maestría.

Ministerio de Educación (1999). Proyecto Educativo Nacional. Coordinación Comisión Promotora Nacional de la Constituyente Educativa. Caracas

Moreno, A. (1945). Ciencia, conocimiento y verdad. Arthopodos, №3

Reyes, M. (2001). Influencia del Rol Familiar en el Rendimiento Estudiantil de los Alumnos de la Tercera Etapa del Ciclo Combinado Arturo Michelena, Calabozo, Estado Guárico. UNERG. Tesis de Grado Maestría.

Ruiz, O y Ispizua, M. (1999). La Descodificación de la Vida Cotidiana. Métodos de Investigación cualitativa. Bilbao. Universidad del Deusto.

Tablante, M (2002). Programa de Escuela para Padres para la Integración de la Familia en el Proceso Educativo de los alumnos de la E.B "Andrés Bello". Tesis de Maestría no publicada. UNERG. Calabozo.

UNESCO (2003). Informe sobre Encuentro Latinoamericano de Educación. Caracas. Venezuela. 